University of Nebraska - Lincoln

DigitalCommons@University of Nebraska - Lincoln

$5-15-2003$

\title{
Spin-wave modes in magnetic nanowires
}

\author{
Ralph Skomski \\ University of Nebraska-Lincoln, rskomski2@unl.edu \\ M. Chipara \\ University of Nebraska-Lincoln, mchipara@utpa.edu \\ David J. Sellmyer \\ University of Nebraska-Lincoln, dsellmyer@unl.edu
}

Follow this and additional works at: https://digitalcommons.unl.edu/physicssellmyer

Part of the Physics Commons

Skomski, Ralph; Chipara, M.; and Sellmyer, David J., "Spin-wave modes in magnetic nanowires" (2003).

David Sellmyer Publications. 37.

https://digitalcommons.unl.edu/physicssellmyer/37

This Article is brought to you for free and open access by the Research Papers in Physics and Astronomy at DigitalCommons@University of Nebraska - Lincoln. It has been accepted for inclusion in David Sellmyer Publications by an authorized administrator of DigitalCommons@University of Nebraska - Lincoln. 


\title{
Spin-wave modes in magnetic nanowires
}

\author{
R. Skomski, ${ }^{\text {a) }}$ M. Chipara, and D. J. Sellmyer \\ Department of Physics and Astronomy and Center for Materials Research and Analysis, \\ University of Nebraska, Lincoln, Nebraska 68588
}

(Presented on 14 November 2002)

\begin{abstract}
Spin-wave modes in magnetic transition-metal nanowires having diameters of about $10 \mathrm{~nm}$ and lengths on the order of $1 \mu \mathrm{m}$ are investigated by model calculations. There are quasicontinuous modes with $k$ vectors parallel to the wires axis and discrete modes with $k$ vectors perpendicular to the wire axis. Due to the small cross section of the wires, the perpendicular modes can be ignored in many cases and the low-temperature behavior of the wires is quasi one-dimensional. Using an analytic approach and exploiting the analogy between micromagnetism and quantum mechanics it is shown that all spin-wave modes with $k$ vectors parallel to the wire axis are localized. The spin-wave localization is a micromagnetic analog to the Anderson localization of conduction electrons due to randomness in less than two dimensions, and, as in the electron analogy, arbitrarily small disorder is sufficient to cause the localization of all modes. (c) 2003 American Institute of Physics. [DOI: 10.1063/1.1558691]
\end{abstract}

\section{INTRODUCTION}

Since Bloch's 1930 article on the temperature dependence of the spontaneous magnetization of ferromagnets, ${ }^{1}$ spin waves (magnons) have attracted much attention in the solid-state community. In particular, spin-wave arguments indicate that there is no long-range isotropic ferromagnetism in two or less dimensions. ${ }^{1-4}$ In Bloch's original approach, the long-wavelength magnetization reduction due to spin waves is proportional to the integral $\int k^{d-3} d k$, which exhibits a long-wavelength divergence for $d \leqslant 2$. On the other hand, experimental structures contain thousands of atoms, which assimilates their behavior to that of bulk ferromagnets. 5 This leads to the question how low-dimensional effects manifest themselves in magnetic nanostructures.

Spin-wave phenomena in ideal cylindrical nanodots and nanowires have recently been studied by a number of authors. $^{7-13}$ There are, for example, spin-wave quantization effects associated with nanoscale dimensions of the magnet. ${ }^{8,13}$ Due to the small radii of electrodeposited wires, less than $5 \mathrm{~nm}$ as compared to lengths of about $1 \mu \mathrm{m},{ }^{5,14}$ there is a pronounced spin-wave quantization for modes whose $k$ vector is perpendicular to the wire direction. Specifically, the lowest-lying quasicoherent mode $(k=0)$, which determines the nucleation field, has an energy significantly lower than that of the curling mode, where $k$ is on the order of $1 / R .^{14,15}$

However, the restriction to perfect nanowires is inadequate in many cases. For example, in real wires the nucleation mode, which can be regarded as a $\omega=0$ spin-wave mode, is localized. The localization is due to deviations from the ideal of an elongated ellipsoid of revolution and accompanied by a coercivity reduction. ${ }^{4,14}$ By contrast, the wellknown coherent-rotation and curling $\operatorname{modes}^{16}$ are delocal-

a) Author to whom correspondence should be addressed; electronic mail: rvdskomski@msn.com ized, that is, they extend throughout the magnet. The same is expected for excited plane-wave modes in bulk magnets. The question therefore arises whether and to what extent excited spin-wave modes in nanowires are subject to localization.

In this article, we analyze the localization behavior of spin waves with $k$ vectors parallel and perpendicular to the wire axis and focus on how imperfections affect the localization behavior.

\section{SPIN-WAVE MODES IN NANOWIRES}

Ignoring damping effects and restricting the consideration to the classical limit, spin waves obey the equation of motion $d \mathbf{M} / d t=\gamma\left(\mathbf{M} \times \mathbf{H}_{\text {eff }}\right)$, where $\gamma$ is the gyromagnetic ratio and $\mu_{\mathrm{o}} \mathbf{H}_{\text {eff }}=-\partial E / \partial \mathbf{M}(\mathbf{r})$ is a local effective field. ${ }^{16-18}$ For homogeneously magnetized ellipsoids of revolution, the effective field is equal to the applied field $\mathbf{H}=H \mathbf{e}_{z}$ plus the anisotropy field $\mathbf{H}_{a}$, and the resonance problem is solved by the diagonalization of a $2 \times 2$ matrix. This uniform or ferromagnetic resonance (FMR) yields resonance frequencies determined by ${ }^{19}$

$$
\omega^{2}=\gamma^{2}\left(H+H_{a x}-H_{a z}\right)\left(H+H_{a y}-H_{a z}\right),
$$

where $\mathbf{e}_{x}$ and $\mathbf{e}_{y}$ correspond to the principal axes of the 2 $\times 2$ matrix. For example, in perfect nanowires aligned parallel to the external magnetic field, this equation degenerates into

$$
\omega=\gamma\left(H+2 K_{\mathrm{eff}} / \mu_{\mathrm{o}} M_{s}\right),
$$

where $K_{\text {eff }}=K_{1}+\mu_{\mathrm{o}} M_{s}^{2} / 4$ is the sum of magnetocrystalline and shape anisotropies. ${ }^{16}$ The electron-spin resonance of these modes is known as spin-wave resonance (SWR), in contrast to the simpler FMR. ${ }^{19}$

The formation of spin waves in a perfect or imperfect magnet costs exchange energy, so that the effective field $H$ $+2 K_{\text {eff }} / \mu_{\mathrm{o}} M_{s}$ entering Eq. (2) must be replaced by ${ }^{16,18}$

$H_{\mathrm{eff}}=\left(2 A / \mu_{\mathrm{o}} M_{s}\right) \nabla^{2}+2 K_{\mathrm{eff}}(\mathbf{r}) / \mu_{\mathrm{o}} M_{s}$. 


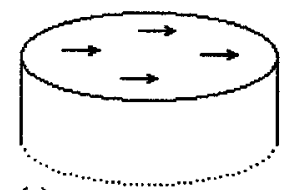

(a)

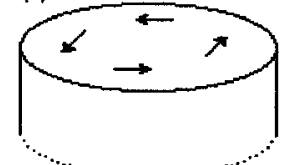

(b)

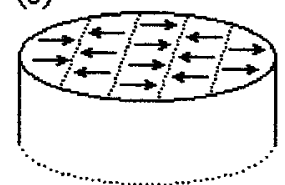

(c)

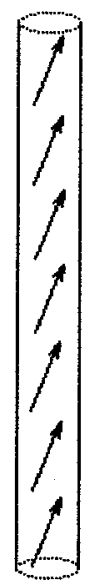

(d)

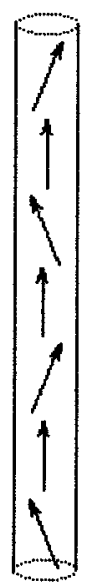

(e)

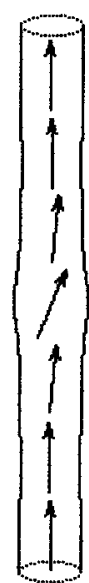

(t)

FIG. 1. Spin-wave modes in nanowires: (a) and (d) coherent mode, (b) curling mode, (c) bulk mode with $k_{\perp}>1 / R$, (e) mode with $k_{\|}>0$, and (f) localized nucleation mode. The bulk modes are essentially superpositions of plane waves. Since the diameter of typical nanowires is much larger than the interatomic distance, there are many excited perpendicular modes, and the finite-temperature magnetization $M_{s}(T)$ is reminiscent of bulk magnets.

Here effective local anisotropy $K_{\text {eff }}(\mathbf{r})$ reflects generally random real-structure inhomogeneities, as discussed, e.g., in Refs. 15 and 20.

Writing the magnetization as $\mathbf{M}=M_{s}\left(1-\mathbf{m}^{2} / 2\right) \mathbf{e}_{z}$ $+\mathbf{m}$, as appropriate in the linear regime, leads us to the consideration of the perpendicular magnetization component $\mathbf{m}(\mathbf{r})$. In cylindrical nanowires having the axis of revolution parallel to $\mathbf{e}_{z}$, it is often useful to write the perpendicular component $\mathbf{m}(x, y, z)$ in cylindrical coordinates, that is, $\mathbf{m}(\rho, \phi, z)$. Figure 1 shows various types of spin-wave modes in nanowires. It is important to distinguish spin waves with wave vectors in the $x-y$ plane $\left(k_{\perp}\right)$ from spin waves with $k$ vectors parallel to the wire axis $\left(k_{\|}\right)$. Figures 1 (a) and 1 (d) show the coherent mode characterized by $k=0$. In perfect elongated ellipsoids of revolution having very small radii, this mode is the nucleation mode $(\omega=0)$. Figure 1(b) shows the curling mode, which is the nucleation mode $(\omega$ $=0$ ) in perfect ellipsoids of revolution having large diameters. In terms of $r$ and $\phi$, this mode is given by $\mathbf{m}$ $= \pm m(r)\left(\cos \phi \mathbf{e}_{y}-\sin \phi \mathbf{e}_{x}\right)$. As it is well known from nucleation-field theory, ${ }^{4,16,21}$ the transition from coherent rotation to curling occurs at an coherence radius scaling as $\left(A / \mu_{\mathrm{o}} M_{s}^{2}\right)^{1 / 2}$. For the ferromagnetic transition metals, this coherence radius is on the order of $10 \mathrm{~nm}$.

In arbitrary systems, coherent rotation and curling are possible as excited states, although they do not necessarily have the character of eigenmodes. Coherent-rotation modes are easily excited by an external magnetic field, whereas the symmetry of the curling mode excludes the interaction with a homogeneous external field. However, curling modes may be excited, for example, by a current flowing through the wire.

In very thin nanowires, curling-type modes can be ignored, ${ }^{5,15}$ and the perpendicular magnetization components obey $\mathbf{M}_{x}=\mathbf{M}_{s} m(z) \cos (\omega t)$ and $M_{y}=M_{s} m(z) \sin (\omega t)$. The function $m(z)$ is given by

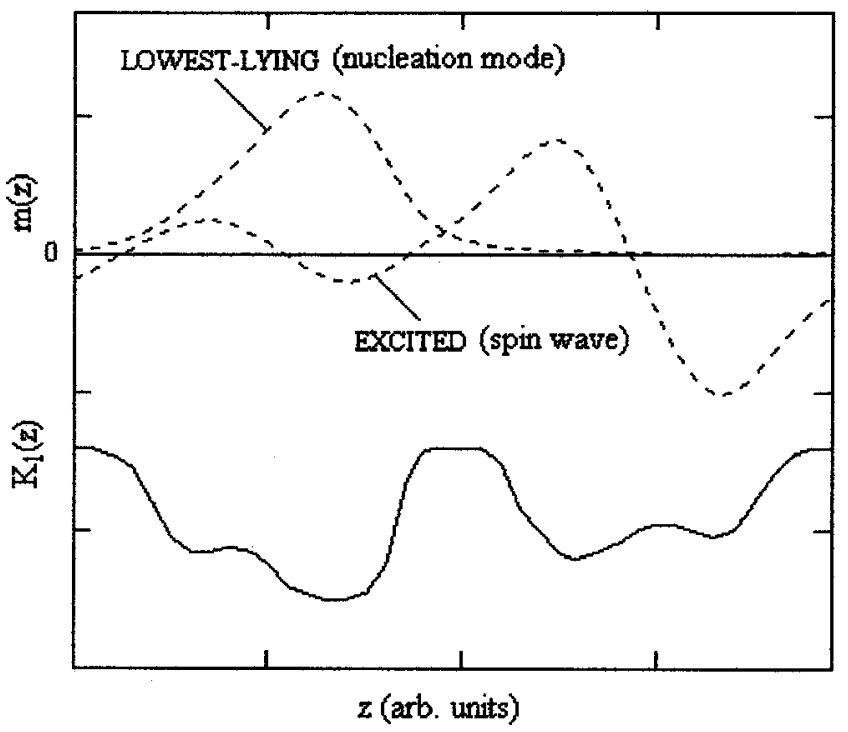

FIG. 2. Localization of spin-wave modes with $k$ vectors along the wire axis. All modes are localized, but the localization length is smallest for low-lying modes.

$$
-2 A \frac{d^{2} m}{d z^{2}}+\left(2 K_{\mathrm{eff}}(z)+\mu_{\mathrm{o}} M_{s} H-\frac{\omega}{\gamma}\right) \quad m=0 .
$$

For $\omega=0$, this equation reduces to the nucleation problem, ${ }^{15,20}$ whereas $d m / d z=0$ reproduces Eq. (2). Mathematically, Eq. (3) is a well-known random-potential eigenvalue problem, which can be solved numerically or by transfer-matrix methods. Figure 2 shows two examples. An interesting point is that all modes are localized. This is expected from the quantum-mechanical analog of a onedimensional electron gas in a random potential. ${ }^{22}$ However, the localization length depends on $\omega$ and is largest for high frequencies. The real-space meaning of the modes is illustrated in Figs. 1(f) and 2.

The spin waves described by Eq. (4) contribute to temperature dependence of the spontaneous magnetization. Treating $k_{\|}$as a continuous quantity we obtain, in analogy with Bloch's original approach, ${ }^{1}$

$$
M_{s}(0)-M_{s}(T) \sim \int \frac{1}{\exp \left(E_{\mathbf{k}} / k_{B} T\right)-1} d k,
$$

where $E_{\mathbf{k}} \approx a^{3}\left(K_{\text {eff }}+A \mathbf{k}^{2}\right)$. Analyzing Eq. (1) yields, after short calculation,

$$
\frac{M_{s}(0)-M_{s}(T)}{M_{s}(0)} \approx \frac{T a \delta_{\mathrm{o}}}{T_{c} R^{2}} .
$$

Here $\delta_{\mathrm{o}}=\left(A / K_{\text {eff }}\right)^{1 / 2}$ is the wall-width parameter, $T_{c}$ is the Curie temperature, and $a$ is the interatomic distance. The magnetization contribution Eq. (6) increases with decreasing wire radius, but since $R$ is much larger than the interatomic spacing, the corresponding magnetization correction remains small. An important exception is the "isotropic" limit, where shape and magnetocrystalline anisotropy contributions cancel $\left(K_{\text {eff }}=0\right), \delta_{0}=\infty$, and the magnetization reduction exhibits the divergence expected for the one-dimensional Heisenberg model. 


\section{DISCUSSION AND CONCLUSIONS}

As discussed e.g., in Ref. 23, the uniform FMR resonance mode Eq. (2) corresponds to the spatially uniform precession of the magnetization, with a magnon wave vector $k$ $=0$. By contrast, Eq. (4) and Fig. 2 describe a nonuniform response to a homogeneous magnetic field. To excite such a mode, the magnetic field must have a nonzero projection onto the mode. In traditional SWR, this projection is realized by surface anisotropies. ${ }^{24,25}$ Due to the randomness of the structural disorder, excitations of the type Fig. 2 lead to a broadening of the resonance lines. However, this broadening is qualitatively different from the inhomogeneous linewidth broadening used, for example, to gauge the homogeneity of an external magnetic field. The difference is the nontrivial involvement of the exchange on length scales comparable to or larger than the wire diameter. A preliminary experimental investigation $\mathrm{Ni}$ nanowires ${ }^{26}$ shows indeed a broadening contribution on the order of magnitude predicted by Eq. (4). In macroscopic wires, the gradient terms in Eqs. (3) and (4) are much less important.

An interesting aspect of the nanowires is the transition from one-dimensional to bulk behavior. Bulk-like behavior is realized by modes with $k_{\perp} \gg 1 / R$, as illustrated in Fig. 1(c), so that there is a natural long wavelength cutoff at $k_{\perp}$ $\approx 1 / R$. At low temperatures, the wires' behavior is governed by long-wavelength magnons, and the behavior is quasi onedimensional, yielding for example Eq. (6). By contrast, at elevated temperatures there is an appreciable number of occupied spin-wave levels with $1 / a<k_{\perp}<1 / R$, and the behavior is bulk-like. The transition temperature is on the order of $A a^{3} / R^{2}$, that is, about $1-4 \mathrm{~K}$ for the considered wires.

In conclusion, we have investigated spin-wave modes in thin transition-metal nanowires. Due to the small radius of wires, the low-lying modes are of the coherent-rotation type, that is, they do not exhibit any special variation in the plane perpendicular to the wire axis. In real wires, the modes are localized, which affects the hysteresis loop and leads to an exchange-stiffness dependence of the resonance field. Lowlying excited states have wave vectors parallel to the wires and lead to a magnetization contribution linear in $T$. This contribution increases with decreasing domain-wall width and, in the isotropic limit its magnitude diverges. When the wavelength becomes comparable to the diameter of the wire, curling-type and higher-order modes interfere, and the magnetic behavior of the wire is bulk-like.

\section{ACKNOWLEDGMENTS}

This research has been supported by DOE, AFOSR, NRI, and CMRA.

${ }^{1}$ F. Bloch, Z. Phys. 61, 206 (1930).

${ }^{2}$ N. W. Ashcroft and N. D. Mermin, Solid State Physics (Holt, New York, 1976).

${ }^{3}$ J. M. Yeomans, Statistical Mechanics of Phase Transitions (Oxford University Press, Oxford, 1992).

${ }^{4}$ R. Skomski and J. M. D. Coey, Permanent Magnetism (Institute of Physics, Bristol, 1999).

${ }^{5}$ D. J. Sellmyer, M. Zheng, and R. Skomski, J. Phys.: Condens. Matter 13, 433 (2001).

${ }^{6}$ J. Shen, R. Skomski, M. Klaua, H. Jenniches, S. S. Manoharan, and J. Kirschner, Phys. Rev. B 56, 2340 (1997).

${ }^{7}$ A. Encinas-Oropesa, M. Demand, L. Piraux, I. Huynen, and U. Ebels, Phys. Rev. B 63, 104415 (2001).

${ }^{8}$ J. Jorzick, S. O. Demokritov, B. Hillebrands, M. Bailleul, C. Fermon, K. Y. Guslienko, A. N. Slavin, D. V. Berkov, and N. L. Gorn, Phys. Rev. Lett. 88, 047204 (2002).

${ }^{9}$ R. Arias and D. L. Mills, Phys. Rev. B 63, 134439 (2001).

${ }^{10}$ S. M. Chérif, Y. Roussigné, P. Moch, J.-F. Hennequin, and M. Labrune, J. Appl. Phys. 85, 5477 (2000).

${ }^{11}$ K. Yu. Guslienko and A. N. Slavin, J. Appl. Phys. 87, 6337 (2000).

${ }^{12}$ U. Ebels, J.-L. Duvail, P. E. Wigen, L. Piraux, L. D. Buda, and K. Ounadjela, Phys. Rev. B 64, 144421 (2001).

${ }^{13}$ Z. K. Wang, M. H. Kuok, S. C. Ng, D. J. Lockwood, M. G. Cottam, K. Nielsch, R. B. Wehrspohn, and U. Gösele, Phys. Rev. Lett. 89, 027201 (2002).

${ }^{14}$ H. Zeng, R. Skomski, L. Menon, Y. Liu, S. Bandyopadhyay, and D. J. Sellmyer, Phys. Rev. B 65, 1344261 (2002).

${ }^{15}$ R. Skomski, H. Zeng, M. Zheng, and D. J. Sellmyer, Phys. Rev. B 62, 3900 (2000).

${ }^{16}$ A. Aharoni, Introduction to the Theory of Ferromagnetism (Oxford University Press, Oxford, 1996).

${ }^{17}$ W. F. Brown, J. Appl. Phys. 30, 62 S (1959).

${ }^{18}$ S. V. Vonsovskii, Magnetism (Wiley, New York, 1974).

${ }^{19}$ C. Kittel, Introduction to Solid-State Physics (Wiley, New York, 1986).

${ }^{20}$ R. Skomski, H. Zeng, and D. J. Sellmyer, J. Magn. Magn. Mater. 249, 175 (2002).

${ }^{21}$ W. F. Brown, Micromagnetics (Wiley, New York, 1963).

${ }^{22}$ P. W. Anderson, Phys. Rev. B 109, 1492 (1958).

${ }^{23}$ M. Farle, Rep. Prog. Phys. 61, 755 (1998).

${ }^{24}$ R. W. Damon and J. R. Eshbach, J. Phys. Chem. Solids 19, 308 (1961).

${ }^{25}$ J. R. Eshbach and R. W. Damon, Phys. Rev. 118, 1208 (1960).

${ }^{26}$ M. I. Chipara, R. Skomski, and D. J. Sellmyer, J. Magn. Magn. Mater. 249, 246 (2002) 